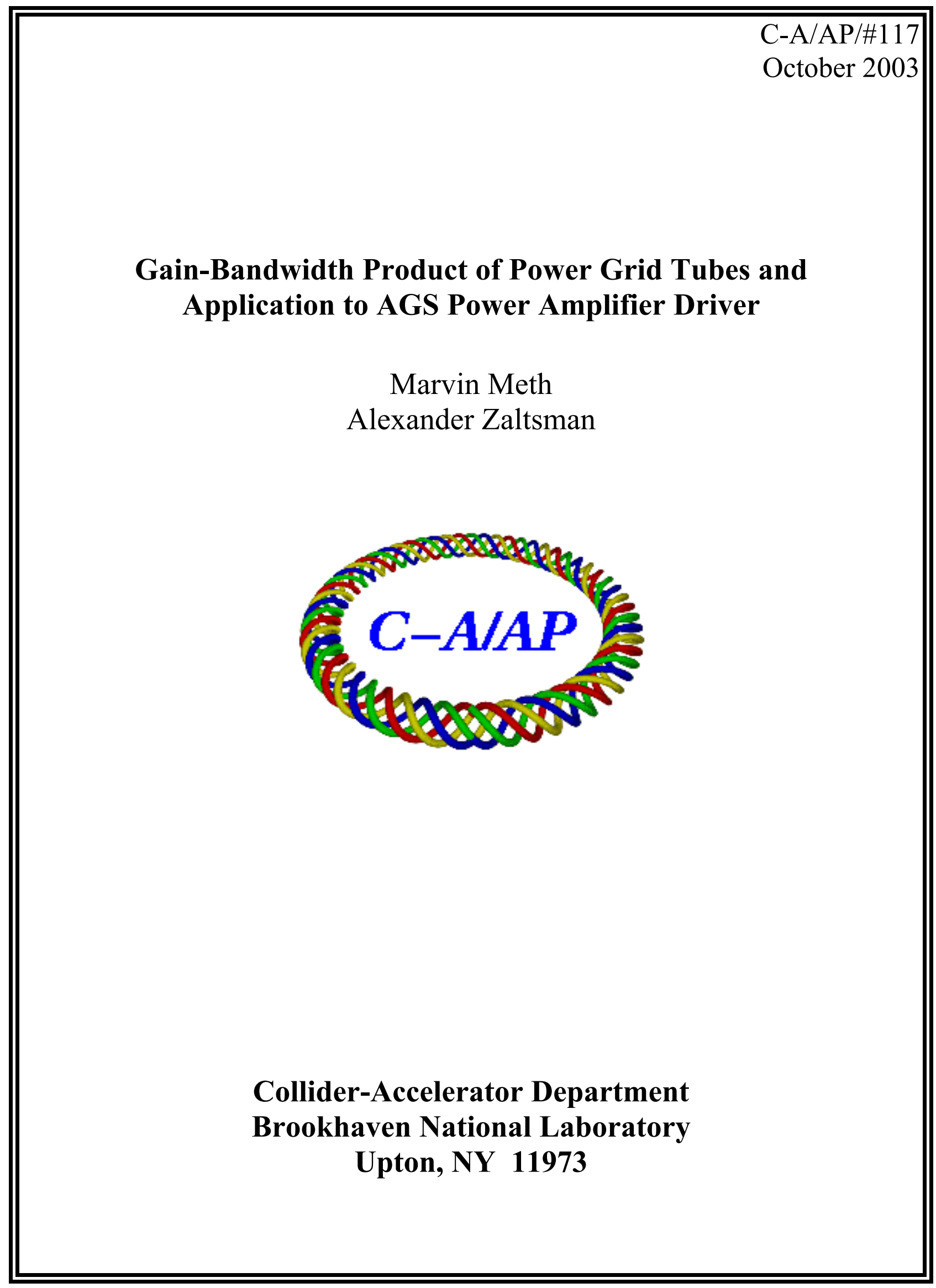




\title{
Gain-Bandwidth Product of Power Grid Tubes and Application to AGS Power Amplifier Driver
}

\author{
Marvin Meth \\ Alexander Zaltsman
}




\section{Introduction:}

The driver for the AGS power amplifier is basically a two-stage feedback amplifier. Each stage consists of four Eimac 4CX350AC tubes connected in parallel, for a total of eight tubes. The second or output stage drives a tuned transformer. The first or input stage is a $\mathrm{RC}$ coupled amplifier. The loading of the first stage is due to the input capacitance of the second stage plus it's own output capacitance. The loading of the preamplifier driver is the input capacitance of the first stage.

The two-stage driver is a wide band rf feedback amplifier. For stability the phase shift of each stage must be limited, requiring a wide bandwidth. To achieve both high gain and wide bandwidth requires that the tubes have a large transconductance $\left(g_{m}\right)$ and a small value of capacitance $\left(\mathrm{C}_{\text {in }}+\mathrm{C}_{\text {out }}\right)$. This can be expressed as a figure of merit [1] defined as $\frac{1}{2 \pi} \frac{g_{m}}{C_{\text {in }}+C_{\text {out }}}$.

\section{Figure of Merit:}

The figure of merit is a measure of the gain-bandwidth product of the stage and is expressed in units of frequency, cycles per second, or 
herz. The division of the figure of merit between gain and bandwidth is in the value of the load resistance. The above definition is for an amplifier with a two-terminal coupling network. For an amplifier with a four-terminal coupling network the figure of merit is defined as $\frac{1}{2 \pi} \frac{g_{m}}{C}$. Where $\mathrm{C}$ is either $\mathrm{C}_{\text {in }}$ or $\mathrm{C}_{\text {out }}$, which ever is the larger.

A survey of the figure of merit for tubes varying in anode dissipation from 19 to 300,000 watts is given in Table I. These tubes were in common use at one time or are presently used in the AGS complex.

\begin{tabular}{c|c|c|c|c} 
Tube Type & $\begin{array}{c}\mathbf{g}_{\mathbf{m}} \\
(\boldsymbol{\mu} \mathbf{~ m h o})\end{array}$ & $\begin{array}{c}\mathbf{C}_{\text {in }} \\
(\boldsymbol{\mu} \boldsymbol{\mu} \mathbf{F})\end{array}$ & $\begin{array}{c}\mathbf{C}_{\text {out }} \\
(\boldsymbol{\mu} \boldsymbol{\mu} \mathbf{F})\end{array}$ & $\begin{array}{c}\text { Figure of } \\
\text { Merit (Mhz) }\end{array}$ \\
\hline \hline 6L6 (19 Watts) & 5,700 & 10 & 12 & 41 \\
807 (25 Watts) & 6,000 & 11 & 7 & 53 \\
814 (50 Watts) & 4,000 & 13.5 & 13.5 & 24 \\
4W300B & 12,000 & 15.7 & 4.5 & 95 \\
4CX350AC & 24,000 & 23.6 & 5.6 & 131 \\
4CX1500B & 30,000 & 85 & 12.8 & 47 \\
4CW2000A & 37,000 & 81.5 & 11.8 & 63 \\
4CW150,000E & 250,000 & 370 & 60 & 93 \\
4CM300,000G & 600,000 & 800 & 84 & 108
\end{tabular}

Table I

Figure of Merit of Beam Power Tetrodes

Two Terminal Coupling 
Discussion:

Examination of Table I reveals that the $4 \mathrm{CX} 350 \mathrm{AC}$ has the highest value of gain-bandwidth product of any available tube. Replacement of the 4CX350AC in the driver by any other tube type would require the employment of a larger number of tubes and or a more complex circuit arrangement.

Paralleling of identical tubes does not affect the gain-bandwidth product of the composite. Nor does it affect the phase characteristics of the composite. A parallel arrangement of identical tubes is characterized by a single (first order) dominat pole with an asymptotic roll-off of $6 \mathrm{db} /$ ocative and a maximum phase shift of $90^{\circ}$.

A series arrangement of $\mathrm{N}$ identical tubes has a reduced bandwidth as compared to a single tube. It is characterized by an $\mathrm{N}^{\text {th }}$ order dominat pole with an asymptotic roll-off of $6 \mathrm{~N} \mathrm{db/octave} \mathrm{and} \mathrm{a} \mathrm{limiting}$ phase shift of $90 \mathrm{~N}$-degrees. A string of three or more identical stages should not be employed in a feedback amplifier due to its excessive phase shift; greater than $180^{\circ}$. 
A series arrangement of three or more stages can be employed provided that one stage has a bandwidth significantly smaller than the other stages. The full gain-bandwidth product of the remaining stages is not utilized, reverting to the reduced gain-bandwidth product of the modified stage.

References:

[1] Terman F.E., Radio Engineer's Handbook; 1943; Page 433. 